\title{
Power Sharing Control of Islanded AC Microgrid Considering Droop Control and Virtual Impedance
}

\author{
Ariel Villalón, Student Member, IEEE, Carlos Muñoz, Rodrigo Aliaga, Student Member, IEEE, Javier Muñoz, \\ Member, IEEE, Marco Rivera, Senior Member, IEEE, Pericle Zanchetta, Senior Member, IEEE
}

\begin{abstract}
Microgrids appear as the key part of the future power systems that include distributed generators, renewable energy, and energy storage. In this paper a decentralized power sharing control scheme that includes droop control with virtual impedances with PI controllers for the voltage and current is proposed for an islanded AC microgrid with two voltage source inverters in parallel that share a residential load. To avoid circulating currents and unbalanced power sharing due to line impedance differences in the microgrid, virtual impedances are added. The proposed control scheme is established in MATLAB/Simulink to prove the proper operation under inductive behavior and mismatches in the line impedances of the microgrid system.
\end{abstract}

Keywords-Microgrid, Power Sharing, Droop Control, Virtual Impedance, Reactive Power Sharing, PI control

\section{INTRODUCTION}

$\mathbf{P}$ Ower systems are currently facing remarkable changes on the way they have been treated for over 100 years with inclusion of renewable energy sources (RES) [1], [2]. This new concept of power systems considers more distributed generation, mainly based on RES, as they are available closer to where the electrical loads are. Nevertheless, there are still technical constraints related to voltage stability and power flow limitations that may lead to large deviations in frequency due to the lack of rotating inertia [3], implying some questions about reliability of such a generation scheme as penetration of RES-based distributed generation grows. These problems are mainly due to the lack of controllability of these distributed generators (DG) to make them more reliable with the possibility of some sections to work isolated as microgrids when faults and contingencies occur in the main grid [4].

Microgrids are mainly small-scale power systems which are formed by DG, EES, and interconnected loads. At the present time, DG systems, which are one of the main actors in microgrids, have a very high controllability and operability degree, thus being able to play a major and decisive role in maintaining the stability of the microgrid [4].

A.Villalón, C. Muñoz, R. Aliaga, J. Muñoz, and M. Rivera are with the Department of Electrical Engineering, Universidad de Talca, Curicó 3344158, Chile (e-mail: avillalon@utalca.cl, carlosmunoz@utalca.cl, roaliaga09@alumnos.utalca.cl,jamunoz@utalca.cl,marcoriv@utalca.cl).

P. Zanchetta is with the Department of Electrical and Electronics Engineering, University of Nottingham. Nottingham NG72RD, U.K. (e-mail: pericle.zanchetta@nottingham.ac.uk).
In grid-connected mode, microgrids can both import energy from and export energy to the main grid. When seen from the point of view of the microgrid, the main grid is considered as stiff; then the microgrid has voltage and frequency levels given by the main grid [5]. In islanded mode, the microgrids operation is not affected by the main grid. Voltage and frequency levels are solely controlled by the DG units, using the voltage source inverters (VSIs) as interface. Thus, power sharing among the DG units that may be embedded in an isolated microgrid has to deal with several aspects, for instance, differences in line impedances, different voltage levels and local loads, among others [6]. One important aspect to deal with, is the presence of circulating currents that may be large and potentially damaging, causing overloaded inverters, reductions in power quality, increasing losses and affecting the efficiency of the system when there are several DG units working in parallel in a microgrid [7]. These circulating currents are caused by mismatches in output voltages, output impedance or among phases in microgrid systems. The latter is not a major issue in a traditional power grid as the line impedances normally reduce these currents, while smaller impedances in microgrids make circulating currents a major issue to take into account [8]. Most of the control techniques are focused on eliminating these circulating currents by power sharing among inverters and considering differences in the line impedances [1], [7]. Differences among the output voltages of the VSCs may cause both active and reactive circulating currents and their effect is dependent on the microgrid's R/X ratio, in addition to the used control methods. To minimize circulating currents, virtual impedance method additionally to droop control can be used [7]. This combination gives good power sharing results and is very applicable for decentralized control of microgrids [9], [10].

The microgrid investigated in this work is depicted in Fig. 1. It includes a residential load as resistive-inductive component, two DG units modeled as ideal DC voltage sources and each connected to an $L C L$ filter for reducing harmonic oscillations. It corresponds to an islanded $\mathrm{AC}$ microgrid.

The goal of this paper is to develop a study of the power sharing among the inverters that are connected to the DG units embedded in an islanded $\mathrm{AC}$ microgrid and the role of virtual impedance for improving the droop control for power sharing among these paralleled inverters. Then, the main contribution of this work lays in the proposal of a complex 


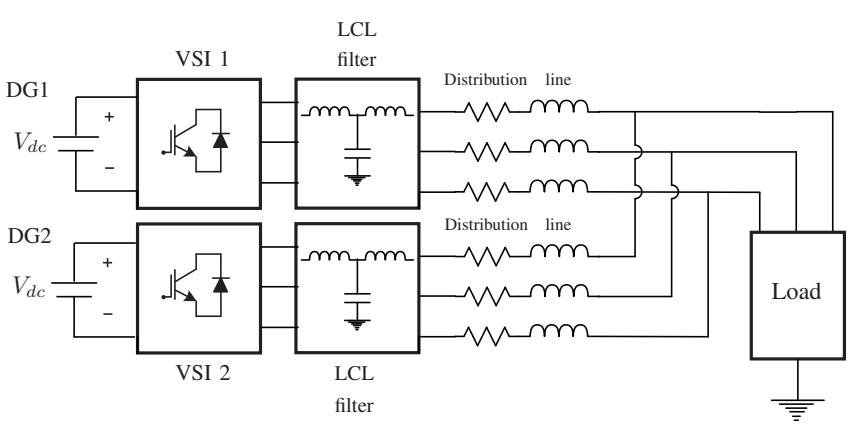

Fig. 1. Topology of the microgrid system

TABLE I

TyPiCAL Line ImPEDANCE VALUES

\begin{tabular}{cccc}
\hline Type of line & $\mathbf{R}(\Omega / \mathrm{km})$ & $\mathbf{X}(\Omega / \mathrm{km})$ & $\mathbf{R} / \mathbf{X}$ (p.u.) \\
\hline Low Voltage Line & 0.642 & 0.083 & 7.7 \\
Medium Voltage Line & 0.161 & 0.190 & 0.85 \\
High Voltage Line & 0.06 & 0.191 & 0.31 \\
\hline
\end{tabular}

virtual impedance that contributes to reducing the voltage drop at the same time that virtual impedance control helps to improve the power sharing among the voltage source inverters in the microgrid system.

\section{Modeling OF THE SYSTEM}

\section{A. System description}

This paper is focused on the islanded microgrid scheme presented in Fig. 1. The system is composed by two DG units interfaced to the islanded AC microgrid with VSIs with LCL output filters with inductors and capacitors. The DG units are modeled as ideal DC voltage sources, not considering their size neither in terms of power or storage capacity.

The microgrid system is composed by distribution lines that follow the impedance values shown in Table I having inductive behavior.

The microgrid system contains one linear load which is shared by two VSIs. The load is resistive-inductive for studying the active and reactive power sharing and is modeled as a constant impedance. The size of the load is chosen to represent a residential load.

\section{CONTROL StRATEgy}

The proposed control scheme for each VSI consists of primary control, an inner-loop control, and virtual-impedance loop. The control scheme is shown in Fig. 2. The details of the control strategy are given in the following subsections.

\section{A. Current control}

Each of the VSIs of the islanded AC microgrid system is controlled to deliver a certain output current through the $L C L$ filter. The current control is the innermost control loop and is the one with the fastest dynamics. It is designed to control the current flowing in the inductor $L_{1}$ of the $L C L$ filter, as shown in Fig. 3.

By applying Kirchoff's voltage law (KVL) on the system in Fig. 3, the following expression is obtained:

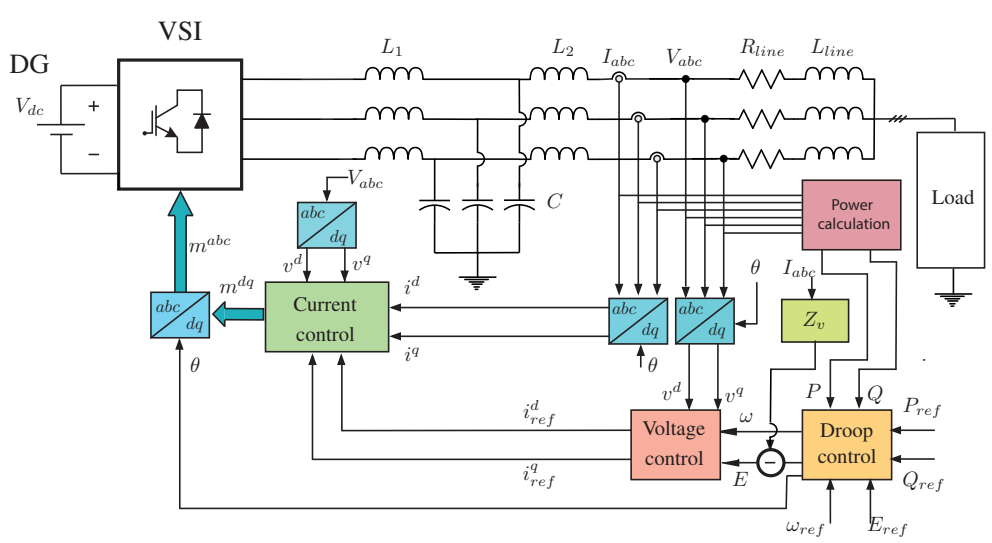

Fig. 2. Proposed control scheme

$$
E_{a b c}=R \cdot i_{a b c}+L_{1} \frac{d i_{a b c}}{d t}+v_{a b c}
$$

Where $E_{a b c}$ is the voltage level before the $L C L$ filter and $v_{a b c}$ is the voltage after the filter. The current through the inverter-side inductor, $L_{1}$, is $i_{a b c}$, and $R$ is the resistor of the grid-side inductor, $L_{2}$.

Considering a balanced system, in the $d q$ reference frame, the equation (1) is as follows:

$$
L_{1} \frac{d}{d t}\left[\begin{array}{l}
i^{d} \\
i^{q}
\end{array}\right]=\left[\begin{array}{l}
E^{d} \\
E^{q}
\end{array}\right]-\left[\begin{array}{l}
v^{d} \\
v^{q}
\end{array}\right]-R\left[\begin{array}{l}
i^{d} \\
i^{q}
\end{array}\right]+\left[\begin{array}{cc}
0 & \omega L_{1} \\
-\omega L_{1} & 0
\end{array}\right]\left[\begin{array}{l}
i^{d} \\
i^{q}
\end{array}\right]
$$

\section{B. Voltage control}

The voltage control loop is for controlling the voltage across the capacitor in the $L C L$ filter. This controller is much slower than the current control loop. Then, any delay related to the current control is neglected. By applying Kirchoff's current law (KCL) on the system in Fig. 3, dynamics of the voltage is given as follows:

$$
i-i_{L}=C \frac{d v_{c}}{d t}
$$

Equation (3) transformed to $d q$ reference frame is as follows:

$$
C \frac{d}{d t}\left[\begin{array}{l}
v^{d} \\
v^{q}
\end{array}\right]=\left[\begin{array}{l}
i^{d} \\
i^{q}
\end{array}\right]-\left[\begin{array}{l}
i_{L}^{d} \\
i_{L}^{q}
\end{array}\right]+\left[\begin{array}{cc}
0 & \omega C \\
-\omega C & 0
\end{array}\right]\left[\begin{array}{l}
v^{d} \\
v^{q}
\end{array}\right]
$$

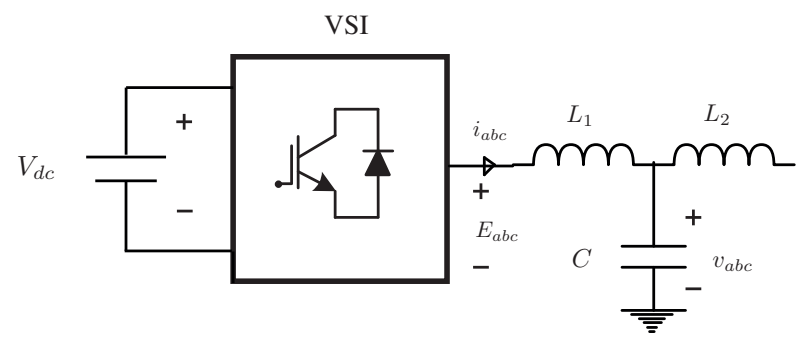

Fig. 3. Three-phase VSI with $L C L$ filter 
In equation (4), $i^{d}$ and $i^{q}$ are the $d q$ components of the current through the inverter-side inductor, $L_{1} \cdot v^{d}$ and $v^{q}$ are the $d q$ components of the voltage across the capacitor in the $L C L$ filter.

1) Natural frequency: to secure sufficient difference in the current and bandwidths of the voltage controllers, the natural frequency of the closed voltage control loop follows the expression:

$$
\omega_{0, v}=\frac{\omega_{0, i}}{10}=\frac{2 \pi f_{s w}}{100}
$$

The value of the natural frequency for the voltage controller, $\omega_{0, v}$, is used to choose the parameters of the PI controllers in the voltage control loop [7].

\section{Droop control}

The primary control of microgrids is the power sharing control which keeps the microgrid voltage frequency and amplitude under control. Each inverter in the microgrid must have an external power loop based on droop control, also called decentralized control. One of the advantages of using this control is that it does not require communication among the inverters of the microgrid, eliminating the limits imposed by physical location of DGs in the microgrid and improving its performance [2].

The impedance values of the distribution lines of the microgrid have influence on droop control.

Considering $P_{A}$ and $Q_{A}$ as the active and reactive powers, respectively, flowing from the source $\mathrm{A}$ (VSI) to $\mathrm{B}$, the grid, $V_{A}$ and $V_{B}$ are the voltage values of these sources, $\delta$ corresponds to the phase-angle difference between the two voltages, $Z=R+j X$ is the interconnection line impedance and $\theta$ is the impedance angle [11].

In the analyzed microgrid system, though it corresponds to a microgrid, the distribution lines are considered as inductive due to the effect of the $L C L$ filter at the output of the VSIs. Thus, in this case, the inductive component of the line impedances is typically much higher, as shown in Table I. Here, the resistive part can be neglected and the power angle $\delta$ in such lines is small, so it can be assumed that $\sin \delta \approx \delta$ and $\cos \delta \approx 1$. The general equations for power flow in the microgrid system can be considered as [11], [12]:

$$
\begin{gathered}
P_{A} \approx \frac{V_{A}}{X}\left(V_{B} \sin \delta\right) \Rightarrow \delta \approx \frac{X P_{A}}{V_{A} V_{B}} \\
Q_{A} \approx \frac{V_{A}}{X}\left(V_{A}-V_{B} \cos \delta\right) \Rightarrow V_{A}-V_{B} \approx \frac{X Q_{A}}{V_{A}}
\end{gathered}
$$

It is very clear from equations (6) and (7) that there is direct relationship between the power angle $\delta$ and the active power $P$ as well as between the voltage difference $V_{A}-V_{B}$ and the reactive power $Q$. With these relationships, the regulation of the microgrid frequency and voltage at the point of connection of the VSI, by controlling the value of the active and reactive powers delivered to the grid is possible. Bearing in mind the
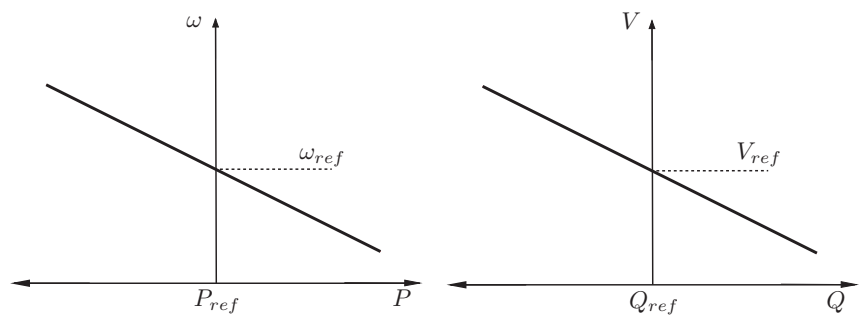

Fig. 4. Frequency and voltage droop characteristics in microgrids with dominant inductive behavior

latter, the equations for droop control for inductive lines are the following [11], [12]:

$$
\begin{gathered}
\omega-\omega_{r e f}=-k_{p}\left(P-P_{r e f}\right) \\
V-V_{r e f}=-k_{q}\left(Q-Q_{r e f}\right)
\end{gathered}
$$

Where $\omega-\omega_{\text {ref }}$ and $V-V_{\text {ref }}$ are the microgrid frequency and the voltage deviations, respectively, from their rated values, and $P-P_{\text {ref }}$ and $Q-Q_{\text {ref }}$ are the variations in the active and reactive powers delivered by the VSI to compensate such deviations [11], [12].

The equations (8) and (9) are represented in the graphs from the Fig. 4.

The $k_{p}$ and $k_{q}$ from equations (8) and (9) represent the gains of the droop control in each case, i.e., the slope of the frequency and voltage droop characteristic or the so called droop coefficients, respectively [12].

Droop coefficients affect the power sharing among the inverters, where larger droop coefficients lead to better power sharing, though increasing them might lead to instability in the system [13]. These droop coefficients are chosen equally in order to equal power sharing among the VSIs in the microgrid system [7].

\section{Virtual impedance control}

Conventional $P / f$ and $Q / V$ droop controls are effective tools for regulating the voltage magnitude and frequency in MV networks, where the lines have a predominant inductive behavior. Then, this method cannot be applied to LV microgrids, where a small mismatching in the microgrid impedance estimation gives as result an inefficient power sharing among the droop controlled DGs. To solve this, the solution consists on introducing virtually the effect of a large inductor which could be used to link the VSI to the AC bus and thereby the line impedance would be predominantly inductive. Thus, the control loop of the VSI is adapted for including this effect [12]. The virtual impedance modifies the VSI output voltage reference as indicated in equation (10), where the modified voltage reference $V_{\text {ref }}$ is obtained by subtracting the virtual voltage drop across the virtual impedance $Z_{v} \cdot I_{\text {output }}$ from the reference value that comes straight from the droop equations $V^{*}[14],[15]$. 


$$
V_{\text {ref }}=V^{*}-v_{v}=V^{*}-Z_{v} \cdot I_{\text {output }}
$$

The observed drop in the $V_{\text {ref }}$ using this technique is one of its main drawbacks [7]. The use of virtual impedance loops is intended to improve the current sharing among the inverters by normalizing the output impedance of them. Nevertheless, as the improvement in the current sharing occurs through redistribution of the current harmonics, virtual impedance loop may result in an increased voltage distortion at the point of common coupling of a microgrid system [16].

Virtual impedances are to keep the voltage within certain limits, to have sufficient reactive power sharing, to provide system damping, to decouple the active and reactive power [17] and they are commonly used in combination with droop control algorithms to improve active and reactive power sharing [7]. In this paper a solution to implement a virtual impedance avoiding the voltage drop is presented, as these virtual impedances normally are considered to be relatively large to obtain predominantly inductive or resistive equivalent output impedance. The solution applied includes a complex virtual impedance. The virtual impedances are designed as resistive-inductive virtual impedances, considering the virtual voltage drop, $\Delta v_{\text {virtual, abc }}$ as follows:

$$
\Delta v_{v i r t u a l, a b c}=R \cdot i_{a b c}+L \frac{d i_{a b c}}{d t}
$$

For implementing the virtual impedance from equation (11) is transformed to $d q$ reference frame, giving the following expressions and assuming a balanced system:

$$
\begin{gathered}
\Delta v_{\text {virtual }, d q 0}=R \cdot i_{d q 0}+L \frac{d i_{d q 0}}{d t}+j \omega L i_{d q 0} \\
{\left[\begin{array}{c}
\Delta v_{\text {virtual }, d} \\
\Delta v_{\text {virtual }, q}
\end{array}\right]=R \cdot\left[\begin{array}{l}
i^{d} \\
i^{q}
\end{array}\right]+L \cdot s\left[\begin{array}{l}
i^{d} \\
i^{q}
\end{array}\right]+\left[\begin{array}{cc}
0 & -\omega L \\
\omega L & 0
\end{array}\right] \cdot\left[\begin{array}{l}
i^{d} \\
i^{q}
\end{array}\right]}
\end{gathered}
$$

Considering the implementation of the virtual impedance from equation (13), low-pass filters are implemented for achieving a voltage less affected by high-frequency oscillations, contributing to keep the voltage drops caused by the virtual impedance within reasonable limits, since the derivative terms and high-frequency components may cause large voltage deviations [7], [15], [17].

\section{E. PI controllers}

The current and voltage controls are implemented using $P I$ controllers in series with the electrical system. The proportional and integrator gains are chosen using the pole placement method [7].

\section{RESUlts}

To prove the proposed control scheme from Fig. 2, the islanded AC microgrid is implemented in MATLAB/Simulink to carry out simulation tests. As it was mentioned, the objective is to investigate the role of virtual impedances on the improvement of the power sharing and on the mitigation of circulating currents.

The parameters used for the simulation are shown in Table II.

As it was explained, the droop control with predominant inductive physical output impedances is used. The simulation is carried out divided into four time slots to show clearly the convenience of implementing the control scheme. For the first three time slots, several virtual impedances are considered as follows:

- 0 - 2 seconds: the droop control is implemented using no virtual impedances.

- 2 - 4 seconds: virtual impedance $1, Z_{v, 1}$, is added to compensate for the difference in physical output impedance among the lines of the microgrid.

- 4 - 6 seconds: three virtual impedances, $Z_{v, 2}, Z_{v, 3}$, and $Z_{v, 4}$, are included to the microgrid system.

- 6 - 10 seconds: a load step-change is made adding $25 \%$ more of the load detailed in II.

Virtual impedance $1, Z_{v, 1}$, is added to the output of $V S I_{1}$ as well as virtual impedance $4, Z_{v, 4}$.

Virtual impedances 2 and $3, Z_{v, 2}$ and $Z_{v, 3}$, are added to the output of $\mathrm{VSI} I_{2}$.

As it can be seen in Fig. 5 (a), the current controller applied to the VSIs that are working in parallel in the microgrid system, works properly showing that there is a tracking of the reference current for the inverter, in this case, $V S I_{1}$, even when a load step-change of $25 \%$ is applied at $t=6 \mathrm{~s}$.

In Fig. 5 (b), the output voltage of the $V S I_{1}$ can be seen, showing that the output voltage is proper. No changes in the output voltage is seen with a load step-change of $25 \%$ at $t=6$ s.

As it was mentioned, for proving that the power sharing control scheme for the islanded AC microgrid system works properly, several virtual impedances were added to the outer control loop of each of the VSIs. These results can be seen in the following pictures:

- One of the main objectives of a proper power sharing control is to avoid the circulating currents in parallel VSIs that share the load of the microgrid system. In Fig. 5

TABLE II

SIMULATION PARAMETERS

\begin{tabular}{c|cc} 
Parameter & Value & Measurement \\
\hline Load power rating & 5.7 & {$[\mathrm{kVA}]$} \\
Nominal voltage (line-to-line) & 400 & {$[\mathrm{~V}]$} \\
Nominal frequency & 50 & {$[\mathrm{~Hz}]$} \\
Inverter side inductor & 500 & {$[\mu \mathrm{H}]$} \\
Inverter side resistance & 0.28 & {$[\Omega]$} \\
Capacitor & 50 & {$[\mu \mathrm{F}]$} \\
Grid side inductor & 200 & {$[\mu H]$} \\
Impedance line 1 & $0.1488+j 0.4969$ & {$[\Omega]$} \\
Impedance line 2 & $0.0930+j 0.3100$ & {$[\Omega]$} \\
Virtual Impedance 1, $Z_{v, 1}$ & $0.0558+j 0.1869$ & {$[\Omega]$} \\
Virtual Impedance 2, $Z_{v, 2}$ & $-0.0279-j 0.0935$ & {$[\Omega]$} \\
Virtual Impedance 3, $Z_{v, 3}$ & $0.0279+j 0.0935$ & {$[\Omega]$} \\
Virtual Impedance 4, $Z_{v, 4}$ & $0.0279+j 0.0935$ & {$[\Omega]$} \\
\hline
\end{tabular}




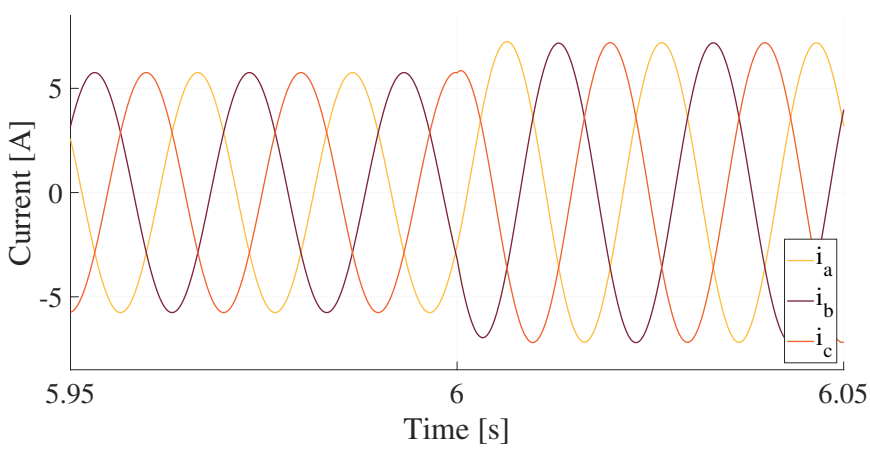

(a)

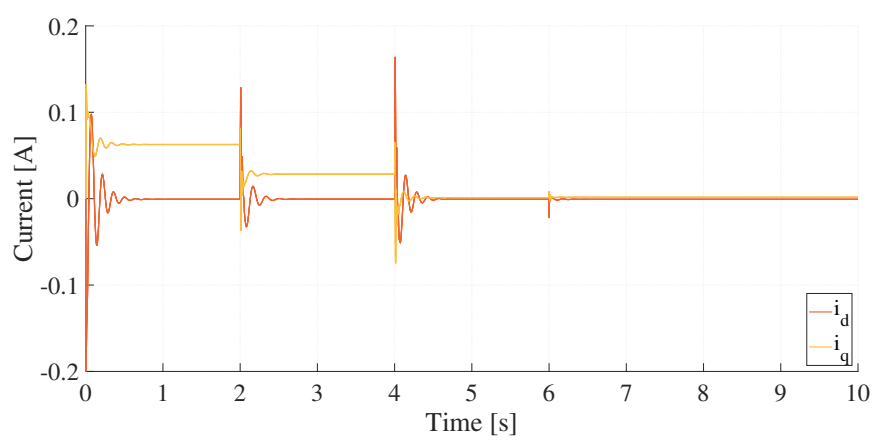

(c)

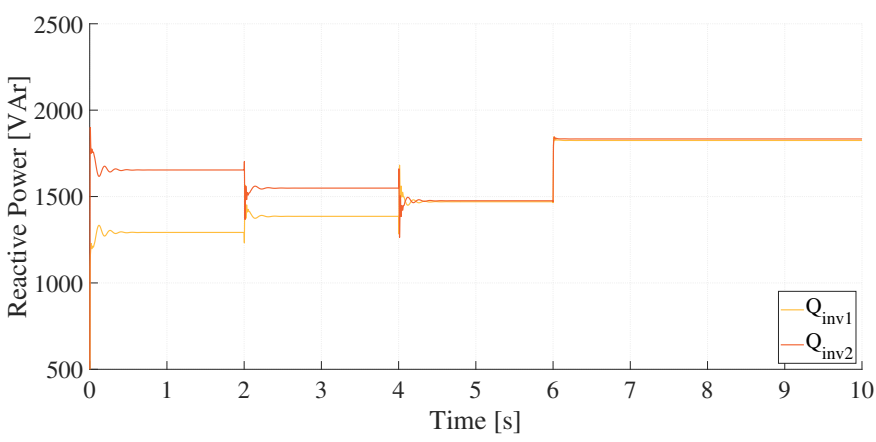

(e)

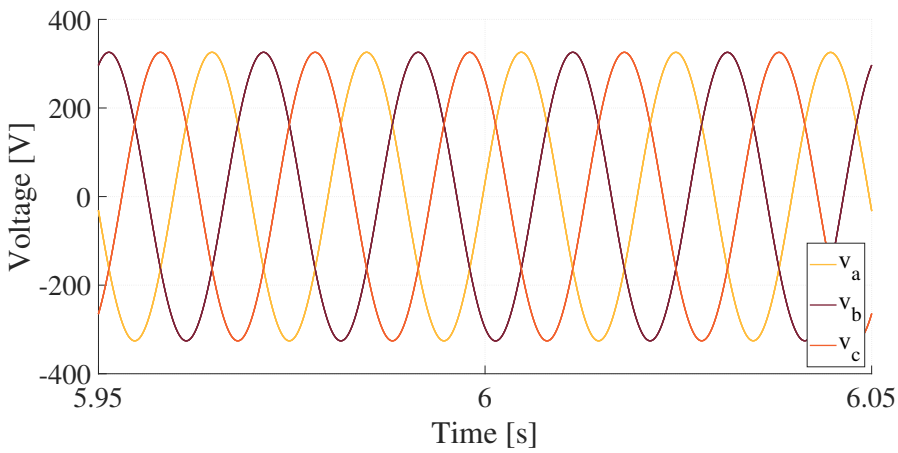

(b)

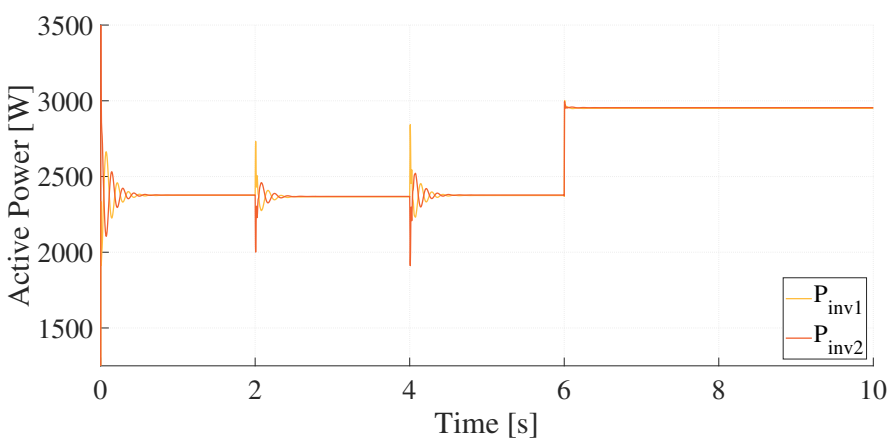

(d)

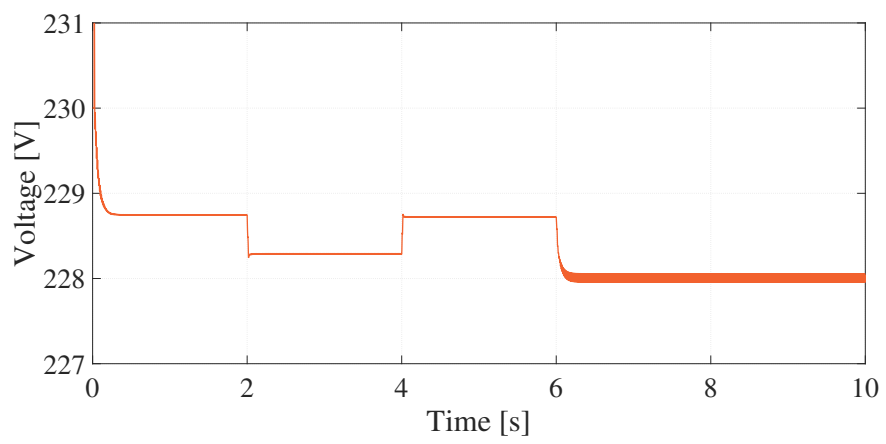

(f)

Fig. 5. Simulation results: (a) output current inverter 1 with load step-change at $t=6$ seconds, (b) output voltage inverter 1 with load step-change at $t=6$ seconds, (c) circulating currents in the microgrid system, $i^{d}$ and $i^{q}$, inverter $1,(\mathrm{~d})$ active power in the microgrid system, (e) reactive power in the microgrid system, (f) load voltage of the microgrid system.

(c) it can be seen very clearly that between $t=0-2$ seconds, the droop control is applied without any virtual impedance added. It remains circulating currents as there are differences in the impedance of the lines. Then, between $t=2-4$ seconds droop control is applied with one virtual impedance added to the outer control loop for the $V S I_{1}$. There still remains some circulating currents in the microgrid system. Afterwards, during $t=4-6 \mathrm{~s}$, three virtual impedances are added to the outer control loop, one for the $V S I_{1}$ and two for the $V S I_{2}$. With these all virtual impedances, it can be seen that no circulating current remains in the system. Additionally, and for proving that the established control scheme works properly, a load step-change is added at $t=6 \mathrm{~s}$. The microgrid system is kept operating with the virtual impedances already mentioned.

- In Fig. 5 (d) the active power sharing is shown. Manifestly, $P$ can be shared accurately using $P / f$ droop control since it is just based on the frequency of the microgrid [18]. Just the transients appear when the virtual impedances are added as mentioned earlier. At $t=6 \mathrm{~s}$, the active power increases as a load step-change of $25 \%$ is added.

- In Fig. 5 (e) the reactive power sharing among $V S I_{1}$ and $V S I_{2}$ is shown. It is clearly seen that at the moment when all the virtual impedances are added $(t=4 \mathrm{~s})$, the reactive power is shared equally by the two inverters in spite of the different physical impedances of the lines of the microgrid. This result shows clearly that the reactive power sharing using $Q / V$ droop control is 
usually substantially affected by the equivalent impedance of the DG and the line impedance [18]-[20]. The virtual impedances are there to equal the impedance among the inverters in order to balance the reactive power sharing in the microgrid system. At $t=6 \mathrm{~s}$, the reactive power increases as a load step-change of $25 \%$ is added.

- Fig. 5 (f) shows that the proposed virtual impedance method from the equation (13) leads to reducing the voltage drop. It can be seen that at $t=6 \mathrm{~s}$, there is a bigger voltage drop as a load step-change of $25 \%$ is added. There is a bigger load to be shared among the two VSIs, so it is reasonable to have this bigger drop in the voltage.

\section{CONCLUSIONS}

In this paper, a droop control method including virtual impedances is proposed to be applied on islanded AC microgrids. Based on the results obtained the following conclusions can be drawn:

- The microgrid system has lines with inductive behavior. As well known, droop control is not able to work properly under this condition, where the reactive power sharing is affected by the physical impedances in the microgrid.

- Virtual impedance control is adequate for enabling the proper reactive power sharing when there are line impedance differences in the microgrid.

- The proposed method for virtual impedance control allows diminishing the voltage drop.

- Adding load step-changes does not change the proper operation of the proposed control scheme.

In order to implement an experimental islanded $\mathrm{AC}$ microgrid with paralleled VSIs that share a load, some considerations should be taken into account: DC sources with galvanic isolation; total power of the load determines the sizing of the several components and of the VSIs. Additionally, from the point of view of the microcontroller, for each of the VSI of the microgrid system, the additional control loops that have to be added may represent a challenge considering the necessary tuning of the controls in order to avoid interferences and thus, to make the power converters work properly.

\section{ACKNOWLEDGEMENTS}

The authors thank the support of the CONICYT/FONDECYT Programme through the Regular 1191028 and the Regular 1160690.

\section{REFERENCES}

[1] M. Zhang, B. Song, and J. Wang, "Circulating Current Control Strategy Based on Equivalent Feeder for Parallel Inverters in Islanded Microgrid," IEEE Transactions on Power Systems, vol. 34, no. 1, pp. 595-605, 2019. [Online]. Available: https://ieeexplore.ieee.org/ document/8449995

[2] X. Wu, C. Shen, and R. Iravani, "A Distributed, Cooperative Frequency and Voltage Control for Microgrids," IEEE Transactions on Smart Grid, vol. 9, no. 4, pp. 2764-2776, 2018

[3] F. Göthner, O.-M. Midtgard, R. Torres-Olguin, and S. D'Arco, "Effect of Including Transient Virtual Impedance in Droop-Controlled Microgrids," in 2018 IEEE International Conference on Environment and Electrical Engineering and 2018 IEEE Industrial and Commercial Power Systems Europe (EEEIC / I\&CPS Europe). Palermo, Italy: IEEE, 2018, pp. $1-6$.
[4] A. H. Yazdavar, M. A. Azzouz, and E. F. El-Saadany, "A Novel Decentralized Control Scheme for Enhanced Nonlinear Load Sharing and Power Quality in Islanded Microgrids," IEEE Transactions on Smart Grid, vol. 10, no. 1, pp. 29-39, 2019.

[5] Z. Peng, J. Wang, D. Bi, Y. Wen, Y. Dai, X. Yin, and Z. J. Shen, "Droop control strategy incorporating coupling compensation and virtual impedance for microgrid application," IEEE Transactions on Energy Conversion, vol. 34, no. 1, pp. 277-291, 2019.

[6] M. H. Andishgar, E. Gholipour, and R.-a. Hooshmand, "An overview of control approaches of inverter-based microgrids in islanding mode of operation," Renewable and Sustainable Energy Reviews, vol. 80, no. August, pp. 1043-1060, 2017. [Online]. Available: http://dx.doi.org/10. 1016/j.rser.2017.05.267

[7] A. B. Holvik, "Virtual Impedance Techniques for Power Sharing Control in AC Islanded Microgrids," Master Thesis, Norwegian University of Science and Technology, 2018. [Online]. Available: https://brage.bibsys.no/xmlui/handle/11250/2562327

[8] R. H. Lasseter, "MicroGrids," in 2002 IEEE Power Engineering Society Winter Meeting. Conference Proceedings. New York, NY, USA: IEEE, 2002, pp. 305-308.

[9] U. B. Tayab, M. A. B. Roslan, L. J. Hwai, and M. Kashif, "A review of droop control techniques for microgrid," Renewable and Sustainable Energy Reviews, vol. 76, no. November 2016, pp. 717-727, 2017. [Online]. Available: http://dx.doi.org/10.1016/j.rser.2017.03.028

[10] X. Yan and Y. Cui, "Control Strategy for Parallel Inverters Based on Virtual Impedance in AC Microgrid," in 2nd IEEE Conference on Energy Internet and Energy System Integration, EI2 2018 - Proceedings. IEEE, 2018, pp. 1-5.

[11] K. De Brabandere, "Voltage and Frequency Droop Control in Low Voltage Grids by Distributed Generators with Inverter Front-End," PhD Thesis, Katholieke University Lueven, 2006. [Online]. Available: https://limo.libis.be/primo-explore/fulldisplay? docid=LIRIAS1730797\&context=L\&vid=Lirias\&search_scope=Lirias\& tab $=$ default tab\&lang $=$ en US\& fromSitemap $=1$

[12] J. Rocabert, A. Luna, F. Blaabjerg, and P. Rodríguez, "Control of Power Converters in AC Microgrids," IEEE Transactions on Power Electronics, vol. 27, no. 11, pp. 4734-4749, 2012. [Online]. Available: https://ieeexplore.ieee.org/document/6200347

[13] C. N. Rowe, T. J. Summers, R. E. Betz, D. J. Cornforth, and T. G. Moore, "Arctan power-frequency droop for improved microgrid stability," IEEE Transactions on Power Electronics, vol. 28, no. 8, pp. 3747-3759, 2013. [Online]. Available: https://ieeexplore.ieee.org/ abstract/document/6362236

[14] J. M. Guerrero, L. Hang, and J. Uceda, "Control of Distributed Uninterruptible Power Supply Systems," IEEE Transactions on Industrial Electronics, vol. 55, no. 8, pp. 2845-2859, 2008. [Online]. Available: https://ieeexplore.iee.org/document/4582449

[15] R. J. Wai, Q. Q. Zhang, and Y. Wang, "A Novel Voltage Stabilization and Power Sharing Control Method Based on Virtual Complex Impedance for an Off-Grid Microgrid," IEEE Transactions on Power Electronics, vol. 34, no. 2, pp. 1863-1880, 2019.

[16] L. Zhou, W. Wu, Y. Chen, J. Jiang, X. Zhou, L. Yang, Z. He, K. Yan, and A. Luo, "Harmonic Voltage Distortion Damping Method for Parallel-Connected LCL-Type Inverters in Islanded Operation," IEEE Transactions on Industrial Electronics, vol. 66, no. 11, pp. 9032-9044, 2019.

[17] X. Wang, Y. W. Li, F. Blaabjerg, and P. C. Loh, "Virtual-ImpedanceBased Control for Voltage-Source and Current-Source Converters," IEEE Transactions on Power Electronics, vol. 30, no. 12, pp. 7019-7037, 2015.

[18] Z. Shuai, S. Mo, J. Wang, Z. J. Shen, W. Tian, and Y. Feng, "Droop control method for load share and voltage regulation in high-voltage microgrids," Journal of Modern Power Systems and Clean Energy, vol. 4, no. 1, pp. 76-86, 2016.

[19] J. M. Guerrero, L. G. De Vicuña, J. Matas, J. Miret, and M. Castilla, "Output impedance design of parallel-connected UPS inverters with wireless load-sharing control," IEEE Transactions on Industrial Electronics, vol. 52, no. 4, pp. 1126-1135, 2005. [Online]. Available: https://ieeexplore.iee.org/document/1490703/

[20] A. Micallef, M. Apap, C. Spiteri-Staines, J. M. Guerrero, and J. C. Vasquez, "Reactive power sharing and voltage harmonic distortion compensation of droop controlled single phase islanded microgrids," IEEE Transactions on Smart Grid, vol. 5, no. 3, pp. 1149-1158, 2014. 